\title{
THE SEDIMENTATION RATE IN RHEUMATIC CARDITIS
}

\author{
BX \\ C. BRUCE PERRY, M.D., M.R.C.P. \\ (From the Winford Orthopaedic Hospital and the University Centre. \\ of Cardiac Research, Bristol General Hospital.)
}

One of the difficulties in the treatment of children with rheumatic carditis is the problem of knowing when the activity of the infection in any given attack has come to an end and it is safe to allow the child to return to an active life. Some time ago the leucocyte count in cases of rheumatic carditis was studjed in the hope of finding some guide as to the quiescence or activity of the infection from the blood picture. It was found that while there was a definite leucocytosis associated with the disease the change was not sufficiently marked to be of value in assessing activity. Since the original observation by $F^{\circ}$ ahraeus in 1917 on the sedimentation rate of the red blood cells much attention has been paid to this phenomenon as an index of activity of infection in various acute and chronic diseases. Kahlmeter $^{6}$ at the Bath Conference in 1928 was one of the first to draw attention to the value of the sedimentation rate in acute rheumatism, and he described cases in which the raised sedimentation rate did not fall to normal until one year after the onset of arthritis. Ernstene ${ }^{3}$ stressed the importance of correcting the sedimentation rate for the degree of anaemia present since a diminution in the total number of red cells, in itself, leads to an increased sedimentation rate. More recently, Åkerrén ${ }^{1}$, Payne ${ }^{7}$, Bach and Gray Hill ${ }^{2}$, and Struthers and Bacal ${ }^{9}$ have shown how closely the sedimentation rate parallels the clinical course of rheumatic carditis in children. These observers formed the opinion that the sedimentation rate was the most delicate index of activity of infection in rheumatic carditis, and that it would prove of value in determining the presence or absence of such activity in doubtful cases: Peterman and Seeger ${ }^{8}$, however, studied the sedimentation rate in a large number of children and found that so great were the variations met with both in health and ill-health that a single determination was of little value in diagnosis and of no value in prognosis. They found that a single normal or slightly altered rate meant little, whereas a single rapid rate must be held to indicate some abnormality.

It is unfortunate that so many methods of performing this test have been described, the result of which is that it is difficult to compare the results of different workers. One of the difficulties in applying the test repeatedly in children, as is necessary in studying the changes during the course of such a disease as rheumatism, is the fact that the majority of the methods necessitate venous puncture, Payne ${ }^{7}$ described a method which 
required 0.4 c.c. of blood and stated that he experienced little difficulty in obtaining this from one stab of the finger pulp. In less experienced hands it has proved difficult to obtain this quantity of blood from a finger prick, especially in the case of younger children. The method adopted in this study has therefore been slightly modified from that described by Payne.

\section{Present method.}

Into a 0.2 c.c. pipette, 0.03 c.c. of isotonic sodium citrate solution is drawn. A finger is cleaned with spirit, congested with a bandage and pricked. Blood is drawn into the pipette until 0.12 c.c. of blood is obtained (making $0 \cdot 15$ c.c. of blood and citrate solution). This is ejected into a small glass bottle and well shaken. Capillary tubing of $0.5 \mathrm{~mm}$. bore is used as a sedimentation tube. From one end of the tube $10 \mathrm{~cm}$. are measured off and the tube filled with blood to this mark. The bottom end of the tube is then sealed with plasticene and the tube stood upright in a lump of plasticene. The level to which the red cells have sunk is read at the end of one hour. It is found that for clinical purposes this gives all the information required. Since it is rare for the anaemia of acute rheumatism to show any very marked decrease in the number of red cells it has not been considered necessary to correct the sedimentation rate as recommended by Ernstene.

An objection to the use of such a small bore capillary tubing is that if the sedimentation rate is rapid the boundary zone between the clear supernatant plasma and the sedimented red cells is in some cases blurred. This only occurs in the minority of cases and only in those in which the rate is rapid where accuracy of reading to 2 or $3 \mathrm{~mm}$. is not essential.

\section{Results.}

In this study 1,043 estimations of the sedimentation rate nave been made on 167 children suffering from rheumatic heart disease. The cases have been divided into five groups. The first group comprises 63 children in whom there was no clinical evidence of active rheumatic infection at the time of examination and there was no loss of weight or increase in physical signs when next seen. On these children 202 observations were made. Only one isolated estimation was made in 22 cases and in all of these the sedimentation rate was under $10 \mathrm{~mm}$. in the first hour. The remaining 41 cases had several estimations made in each case (180 in all) over varying periods of time, the average length of time being about eight months. In 3 cases a sedimentation rate over $20 \mathrm{~mm}$. in the first hour was found, in 2 of these it was associated with fever and a ' cold,' but in one case no cause could be found for the abnormal rate. There were also 27 readings over $10 \mathrm{~mm}$. in the first hour; one of these was a child suffering from a ' cold' and eleven occurred in the same boy who was doing well, gaining weight, with no other evidence of activity, and with no change in physical signs either at the time or later; in the remaining 15 readings no cause for the abnormal rate could be found. Thus over 85 per cent. of the observations made on the sedimentation rate in apparently quiescent cases of rheumatic carditis were under $10 \mathrm{~mm}$. in the first hour. It might be claimed that some of the abnormal rates not otherwise explained might indicate a mild relapse of rheumatism or an observation made at the end of a relapse. In view of the subsequent progress of the case this is unlikely. Since so many factors are known to produce an increase in the sedimentation 
rate it is hardly surprising that nearly 18 per cent. of apparently quiescent cases showed abnormal rates, and it must be recognized that a high sedimentation rate in a child with rheumatic heart disease does not necessarily indicate active infection, although, as will be shown later, it should lead to a very careful investigation of the case to exclude such activity.

The cases manifesting evidence of active rheumatism at some time or other during the period of observation have been divided into four groups. The first group is composed of those cases showing chorea as the only sign of rheumatism and with no carditis; the second group consists of those children who had chorea with carditis. In the third and largest group have been considered those cases observed in attacks or relapses of carditis, without chorea as an initial feature, in whom the attack ran a ' monocyclic' form (Homer Swift). The fourth group comprises the children exhibiting polycyclic types of relapses or attacks.

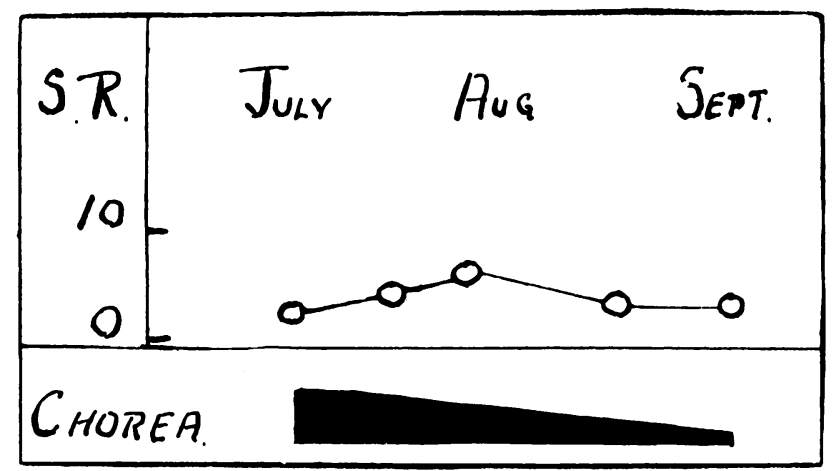

Fig. 1. Case 1 .

Thirteen children fell into the first group and on these cases 36 observations were made. All these children had active chorea at the time of the first observation with no evidence of carditis. In one of these the sedimentation rate was 26 at the first observation and gradually fell to 8 in two months. One other case showed a rate of 12 at the first observation which fell to 1.5 in four weeks. With these exceptions the remaining 11 children showed normal sedimentation rates throughout the period of observation. This finding of a normal sedimentation rate in uncomplicated chorea agrees with the observations of $\mathrm{Warner}^{10}$, Struthers and Bacal ${ }^{9}$, and and Faxen ${ }^{4}$. The cause for this is not clear since on all other grounds it is generally agreed that chorea is an active rheumatic manifestation. The following case is typical of the group:-

Case 1.-A girl, aged $13_{\frac{9}{2}}^{9}$, had a previous attack of chorea 18 months ago. July 11, 1933: recurrence of chorea; no abnormal cardiac physical signs. July 17 : admitted to hospital; chorea steadily improved and was almost recovered on discharge to convalescent home on September 12. No signs of carditis ever detected (fig. 1). 
In the group of cases of chorea with carditis a different state of things is found. This group comprised 16 cases and on these 87 observations were made. Two cases of chorea with apparently active carditis showed sedimentation rates under 10, but apart from this all cases had sedimentation rates over 10 and with three exceptions over 20. One case is of interest in that the child was first seen with chorea but with no signs of carditis, the sedimentation rate was 5 . When seen later definite signs of carditis were present and the sedimentation rate was now 16, during convalescence it fell again to 5 . The highest sedimentation rate observed was 46. Thus in the majority of cases of chorea with carditis a high sedimentation rate is shown in contrast to uncomplicated chorea. The following case illustrates these features:-

Case 2.-A girl, aged $12 \frac{1}{2}$, with an old cardiac lesion dating from an attack of chorea at the age of $9 \frac{1}{1}$. August 22 : quiescent, sedimentation rate 4 . November 10 : mild chorea, no increase in cardiac physical signs, sedimentation rate 21 . November 24: chorea improved, sedimentation rate 13. December 8: no obvious chorea, heart unchanged, sedimentation rate 7 . December 29 : better, sedimentation rate 4. January 19 : going on well, sedimentation rate 8 . January 29 : sedimentation rate 5. February 12: sedimentation rate 2. March 1: sedimentation rate 2, steady improvement (fig. 2).

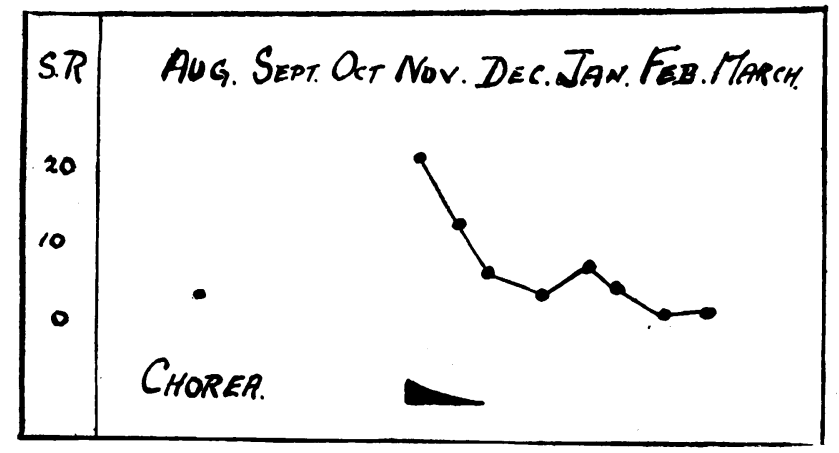

Fig. 2. Case 2.

The third group of cases observed in an acute monocyclic attack or relapse comprised 47 children observed in 49 attacks and on whom 235 observations were made. In this group every case in the attack showed a sedimentation rate over 20. The fastest rate noted in the attack was 60 and the lowest 21. The time taken for the sedimentation rate to return to normal varied from one month to four mouths. Some cases were only seen late in the attack when improvement was obvious, twelve of these showed sedimentation rates under 20 and in 2 cases not seen till 5 or 6 weeks after the attack the sedimentation rate was normal (i.e. under 10). It is interesting to note that two cases during convalescence and after the sedimentation rates had fallen to normal limits developed mild chorea which produced no effect on the sedimentation rate. The following case illustrates this :- 
Gase 3.-A girl, aged $8_{\frac{7}{2}}^{\frac{7}{2}}$, seen on January 2, 1934, with a history of limb pains last September, not well since, many subcutaneous nodules found on both elbows and on the spines of the vertebrae, slight cardiac enlargement present with a loud apical systolic murmur, sedimentation rate $20 \mathrm{~mm}$. January 9 : slight chorea. January 11 : marked chorea, cardiac signs unchanged, sedimentation rate 2 . January 19 : sedimentation rate 2. January 23: chorea persists, no cardiac enlargement, murmur very soft. January 26: sedimentation rate 2.5. February 13: nodules decreasing, cardiac signs unchanged, chorea much less, sedimentation rate 4. February 21 : discharged to Torquay (fig. 3).

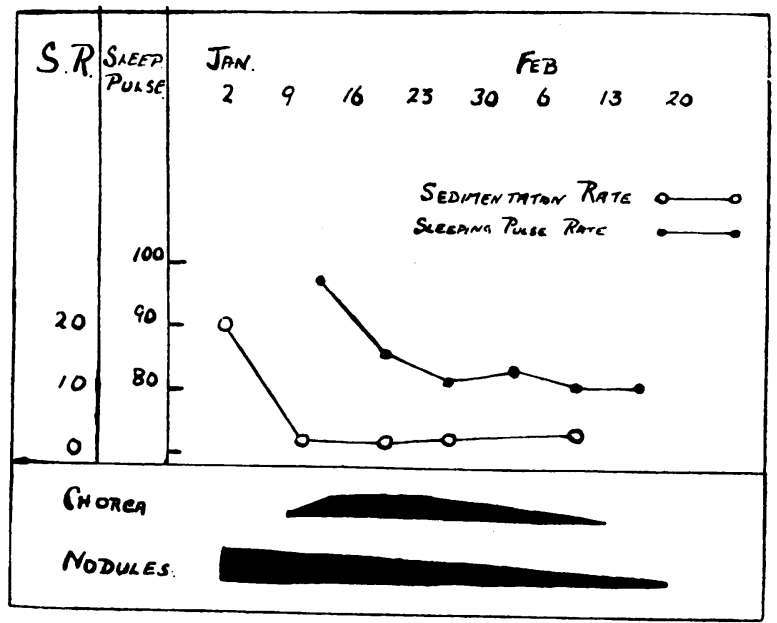

FIg. 3. Case 3.

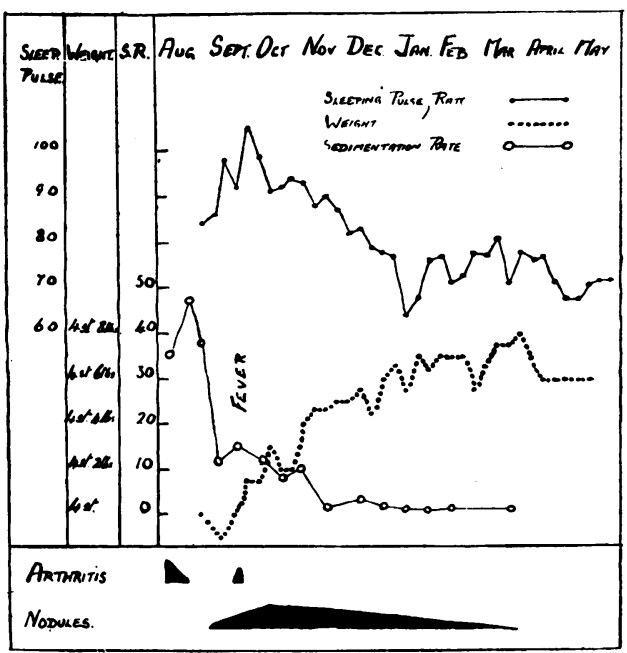

Fig. 4. Case 4.

These cases were observed for varying times throughout convalescence and after recovery from the attack. There were 11 occasions during convalescence when the sedimentation rate was found to be over 10, the highest figure noted being 15 in one case. The following case is typical of the group:- 
Case 4.-A boy, aged 10, with an old heart lesion dating from chorea at the age of three. Several relapses had occurred since then. Considerable cardiac enlargement found with systolic and mid-diastolic murmurs at the apex. June and July: going on well, no evidence of activity, sedimentation rate 4 and 3. August 11: one week's history of pains, finger joints red and swollen, sedimentation rate 35 . August 15 : knees swollen. August 21 : sedimentation rate 47. August 31 : sedimentation rate 37. September 11: loss of weight, otherwise improved, small nodules over metacarpophalangeal joints, knees and ankles, sedimentation rate 13. September 25: nodules larger, cardiac signs not appreciably changed, sedimentation rate 15 . September 27 : pain and swelling of finger joints lasting two days. October 9 : nodules on occiput and spine in addition to others, sedimentation rate 12 . October 23: better, nodules unchanged, sedimentation rate 9. November 6: general condition improved, nodules persist, sedimentation rate 10. November 24: improving, nodules unchanged, sedimentation rate 2. December 19 : general condition improved, sedimentation rate 4. January 1: nodules smaller and fewer, sedimentation rate 2. January 15: nodules decreasing, sedimentation rate 1. January 29: nodules decreasing, improving rapidly, sedimentation rate 1. February 20 : nodules decreasing, better, sedimentation rate 2. March 20 : no nodules, sedimentation rate 1.5 (fig. 4).

In the fourth group of children observed in attacks or relapses running a polycyclic course are 27 children, with 30 attacks, and on whom 483 observations were made. In no case during activity was the sedimentation rate under 10, the highest rate found being 68 , and the lowest 15 . In one case the abnormal rate persisted for 14 months. At the end of this time the child was discharged home but had to be re-admitted almost immediately as she developed an obvious relapse with acute polyarthritis shortly after discharge. Apart from the abnormal sedimentation rate the only evidence of activity was a sleeping pulse rate varying between 85 and 95 . With further time spent in hospital the sedimentation rate finally fell to within normal limits. Although in most cases the persistently abnormal sedimentation rate gave a clue to the smouldering infection which was not apparent clinically, yet in some the rate fell to below 10 during remissions. This is shown by case 5 .

Case 5.-A boy, aged 15, seen on November 11, 1933, with pains in arms and legs and joints, with swelling of the joints. November 17: admitted to hospital, no definitely abnormal cardiac physical signs, fleeting swelling and pain in all joints, marked erythema marginatum over the whole body. December 6 : sedimentation rate 5. December 15: sedimentation rate 12. December 24: much improved, erythema persists. December 29: rise of temperature, relapse of joint pains, apical systolic murmur, sedimentation rate 34. January 3, 1934: nodules on flexor tendons of hands, pains better. January 4: sedimentation rate 23. January 11 : sedimentation rate 35. January 19 : sedimentation rate 9. January 23: fresh rise of temperature with precordial pain and pericardial rub. January 26: sedimentation rate 49 . January 31 : fresh rise of temperature with joint pains. February 13: nodules still present, no cardiac enlargement, no apical systolic murmur, no joint pains or rash, sedimentation rate 27. March 5: all physical signs disappeared, sedimentation rate 2. March 16: steady improvement, sedimentation rate 2,- no further relapse except for occasional erythema. April 30 : sedimentation rate 1.5 (fig. 5).

In one of the cases observed through two attacks the sedimentation rate was abnormal over a month before any clinical evidence of a relapse was found. During convalescence and after recovery four of the cases showed transient sedimentation rate over 10 and in one this was over 20, but this was associated with a transient unexplained fever. There was no 
associated clinical relapse of rheumatism. In two cases developing congestive cardiac failure under observation the sedimentation rate became normal, although the disease was obviously active, and in one case that recovered the rate became raised again as the oedema cleared. This

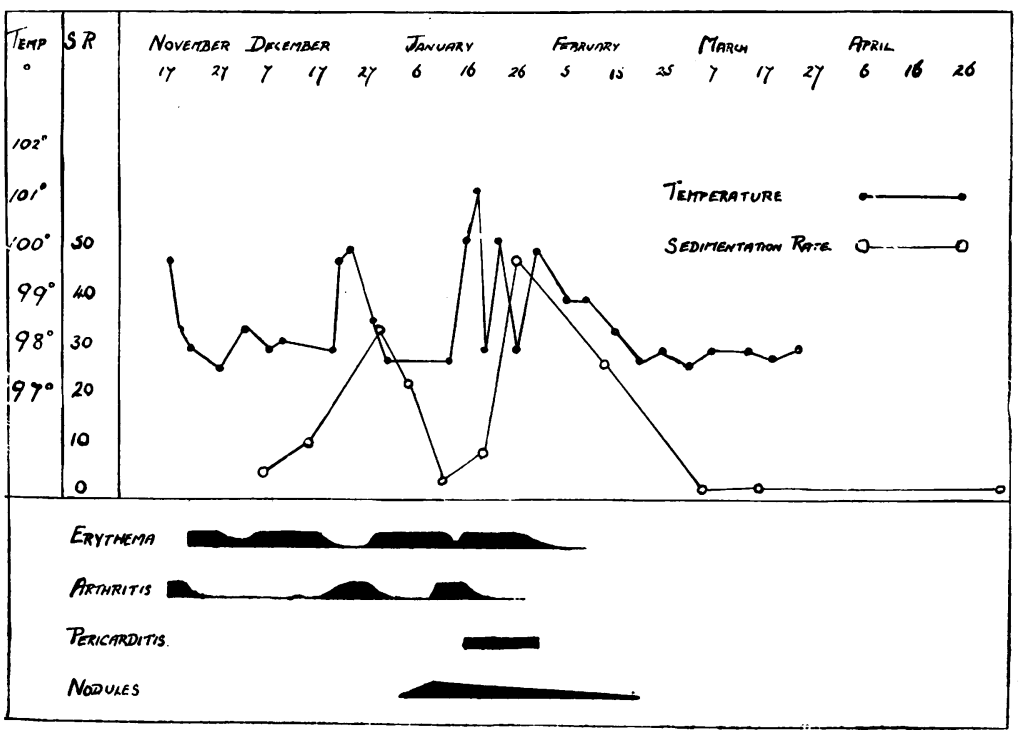

Fig. 5. Case 5.

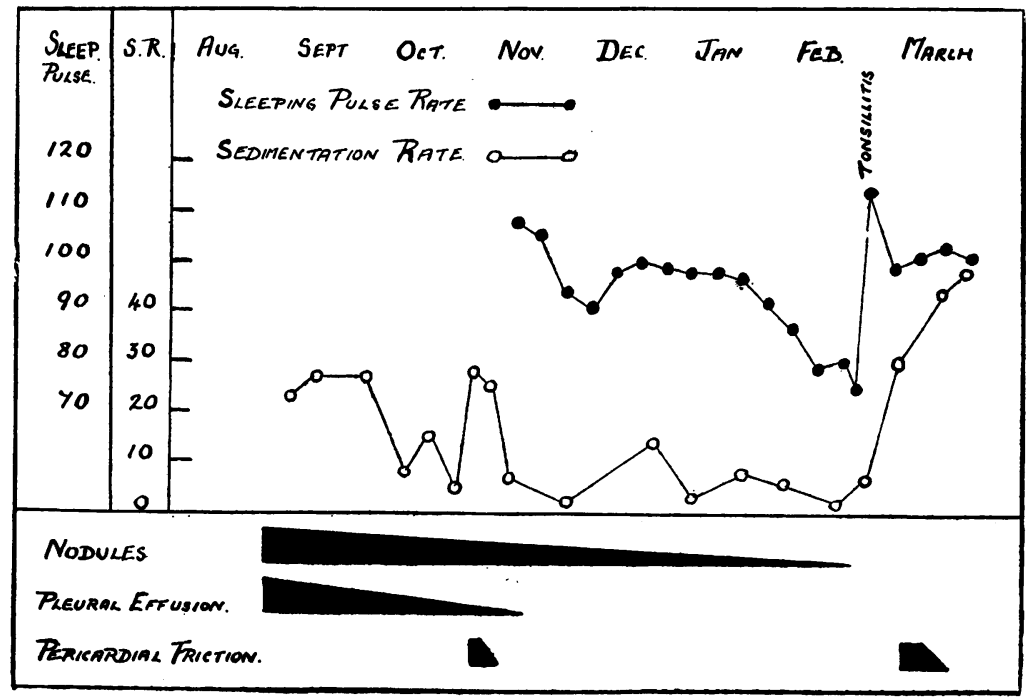

Fig. 6. Case 6.

anomaly is presumably to be explained by a change in the plasma proteins coincident with the oedema, since there is much evidence that these play an important part in regulating the sedimentation rate of the red blood cells. Case 6 illustrates the features of this group. 
Case 6.-A girl, aged 13, seen in August, 1933, with an acute relapse of carditis and left sided pleural effusion together with many subcutaneous nodules; sedimentation rate 23. Slow improvement with fall of sedimentation rate to 5 on October 17 . October 23: relapse with pericarditis, sedimentation rate 29. Gradual improvement followed, sedimentation rate falling to 7 on November 6. Gradual improvement with fall in sleeping pulse rate and gain in weight, and by February 19 the nodules had practically disappeared. February 21 : haemolytic streptococcal tonsillitis. This was followed by rapid loss of weight. March 1: an aortic diastolic murmur noted for the first time; sedimentation rate 30. March 8: pericarditis, friction persisting for more than 10 days. March 15: sedimentation rate 44. March 20: clinically improved, sedimentation rate 49 . The child was removed from hospital against advice and died 14 days later (fig. 6).

\section{Discussion.}

The high sedimentation rate found in acute rheumatism is in no way a specific reaction since many other infections, both acute and chronic, show a similar change. The absence of any constantly high sedimentation rate in chorea uncomplicated by carditis is puzzling. Payne (personal communication) has suggested that this finding is due to the fact that the cases with chorea have not come under observation until late in the disease when the chorea is a residual phenomenon. The two cases who developed chorea during convalescence from an attack of rheumatism with no change in the sedimentation rate suggest that this is not the true explanation. It was hoped that the sedimentation rate might provide a clue to those smouldering cases which show no gross clinical evidence of activity, but deteriorate rapidly on being set free from restraint and treatment. In some cases this is so and it is clear from this study that any rheumatic child showing a persistently abnormal sedimentation rate should be regarded as having active disease unless some other cause for the blood change can be found. Unfortunately the reverse does not hold true-namely that any single normal sedimentation rate excludes the possibility of a smouldering activity, but if the sedimentation rate is persistently low it is unlikely that the rheumatic process is active. This observation raises an interesting point, whether some of these cases should be regarded as exhibiting exacerbations of a long drawn out infection or rather acute reinfections during convalescence.

Observations on the correlation between the sedimentation rate and the development and duration of subcutaneous rheumatic nodules have also been made. In all, 16 cases had subcutaneous nodules. As is usual the nodules developed at the end or late in the attack and in 9 of the cases they appeared when the sedimentation rates had fallen to between 10 and 20, in 6 the sedimentation rate was over 20 at the time that the nodules first appeared; in the remaining case which was not seen until the nodules had appeared the sedimentation rate was 6 . On the other hand, in no case, in which the development of nodules was observed, did fresh nodules appear after the sedimentation rate had fallen below 10. There is one possible exception to this statement and that is case 5. In this instance nodules 
developed seven weeks after the first attack of rheumatism but at the height of a relapse and between the two acute phases the sedimentation rate had fallen to normal. Whether the nodules should be related to the initial attack or to the exacerbation, it is difficult to say but they persisted through a further exacerbation, lasting in all just over two months.

Thus these observations confirm previous reports of other workers that an acute attack or relapse of rheumatic carditis is associated with a rapid sedimentation rate of the red blood cells. This abnormality of the blood returns to normal after a variable period of convalescence, and it is clear that no case can be regarded as recovered from the acute phase of the disease in which the sedimentation rate remains high. The sedimentation rate is altered early in the course of the disease and may be abnormal before any clinical signs of a rheumatic relapse are evident. On the other hand, in some cases running a protracted polycyclic course the sedimentation rate may return to normal and yet obvious clinical signs of activity recur within two or three weeks. Whether the sedimentation rate should be regarded as the true index of activity and the apparent exacerbation as actually a fresh infection, is a difficult point to determine, but from the fact that such cases present no clinical differences from those in which the sedimentation rate remains abnormal throughout makes it appear unlikely. At the same time in certain cases the abnormal sedimentation rate may be the only sign of activity of the infection and if such cases are followed up clear evidence of active disease appears.

\section{Conclusions.}

(1) Acute rheumatic carditis is always associated with a high sedimentation rate.

(2) Chorea uncomplicated by carditis may show a normal sedimentation rate.

(3) The sedimentation rate is an accurate index of active infection in rheumatic carditis.

(4) While active carditis is unlikely in the presence of a normal sedimentation rate, repeated observations are essential to confirm the absence of infection in cases running a polycyclic course.

(5) Subcutaneous nodules appear late in the course of the disease as judged by the sedimentation rate and may persist after complete quiescence of the disease is established.

(6) With the onset of congestive cardiac failure in acute rheumatic carditis the sedimentation rate returns to normal.

I am indebted to the Colston Research Society for a Medical Fellowship during the tenure of which this work was carried out. 


\section{REFERENGES.}

1. Åkerrén, Y., Acta. paediat., Uppsala, 1931, X, 473.

2. Bach, F., \& Gray Hill, N., Lancet, Lond., 1932, i, 75.

3. Ernstene, A. C., Am. J. Med. Soc., Philad., 1930, CLXXX, 12.

4. Faxen, N., Rev. franc. de pediat., Paris, 1934, IX, 809.

5. Gray Hill, N., Brit. J. Child. Dis., Lond., 1932, XXIX, 181.

6. Kahlmeter, G., Rheumatic Diseases (Bath Conference), Lond., 1928, 219.

7. Payne, W. W., Lancet, Lond., 1932, i, 74.

8. Peterman, M. G., \& Seeger, S. J., Am. J. Dis. Child., Chicago, 1929, XXXVII, 693.

9. Struthers, R. R., \& Bacal, H. L., Can. Med. Ass. J., Montreal, 1933, XXIX, 470.

10. Warner, E. C., Proc. Roy. Soc. Med., Lond., 1934, XXVII, 963. 